\title{
Upgrade of the CMS Tracker with tracking trigger
}

This article has been downloaded from IOPscience. Please scroll down to see the full text article.

2011 JINST 6 C12065

(http://iopscience.iop.org/1748-0221/6/12/C12065)

View the table of contents for this issue, or go to the journal homepage for more

Download details:

IP Address: 137.138.124.233

The article was downloaded on 26/07/2012 at $16: 16$

Please note that terms and conditions apply. 
Topical Workshop on Electronics for Particle Physics 2011, 26-30 SEPTEMBER 2011, VIENNA, AUSTRIA

\section{Upgrade of the CMS Tracker with tracking trigger}

\section{Abbaneo ${ }^{1,2}$}

CERN,

CH-1211, Geneve 23, Switzerland

E-mail: Duccio.Abbaneo@cern.ch

ABSTRACT: The planned upgrades of the LHC and its injector chain are expected to allow operation at luminosities around or above $5 \times 10^{34} \mathrm{~cm}^{-2} \mathrm{~s}^{-1}$ sometimes after 2020 , to eventually reach an integrated luminosity of $3000 \mathrm{fb}^{-1}$ at the end of that decade. In order to fully exploit such operating conditions and the delivered luminosity, CMS needs to upgrade its tracking detectors and substantially improve its trigger capabilities. To achieve such goals, R\&D activities are ongoing to explore options and develop solutions that would allow including tracking information at Level-1. Some of the options considered are reviewed, discussing their potential advantages and disadvantages.

KEYWORDS: Trigger concepts and systems (hardware and software); Si microstrip and pad detectors; Particle tracking detectors (Solid-state detectors)

\footnotetext{
${ }^{1}$ Corresponding author.

${ }^{2}$ On behalf of the CMS Tracker Collaboration.
} 


\section{Contents}

1 The CMS Tracker at the high-luminosity LHC 1

2 Requirements for the strip tracker upgrade $\quad 2$

2.1 Tracking information at Level-1 2

3 Implementation of the tracking trigger 3

3.1 Modules with $\mathrm{p}_{T}$ discrimination 3

3.2 The " $2 \mathrm{~S}$ " module 5

3.2.1 The "PS" module 5

3.2.2 The "VPS" module 6

4 Evaluation of tracker options and geometries $\quad 7$

5 Tracking trigger: from stubs to tracks 9

$\begin{array}{lll}5.1 & \text { Hierarchical processing in FPGA } & 9\end{array}$

5.2 Parallel processing in Associative Memories 9

6 Conclusions 9

\section{The CMS Tracker at the high-luminosity LHC}

The increase of the LHC luminosity well above its original design figure of $10^{34} \mathrm{~cm}^{-2} \mathrm{~s}^{-1}$ requires a substantial upgrade of the CMS tracking system, to cope with much more demanding requirements and to implement additional functionality.

The pixel detector is the first part of the tracker that will show limitations in high-rate and therefore will be upgraded already in the middle of this decade. The new detector [1] will feature 4 barrel layers and 3 forward discs, yielding on average one more spatial point measurement per track compared to the present system, in the whole acceptance range. Optimized engineering of mechanics and services, together with two-phase $\mathrm{CO}_{2}$ cooling, will provide a substantial reduction of material in the tracking volume, while upgraded front-end ASICs will enhance the robustness of the system at high rate. The new pixel detector together with the outer strip tracker will provide optimal tracking performance to CMS through 2020.

After the upgrade of the accelerator complex foreseen for the beginning of the next decade, the LHC is expected to produce an instantaneous luminosity of $5 \times 10^{34} \mathrm{~cm}^{-2} \mathrm{~s}^{-1}$, that will be sustained for a large fraction of each fill through luminosity levelling. The quoted luminosity corresponds to approximately 100 pileup events per bunch crossing if the operating frequency is $40 \mathrm{MHz} .{ }^{1}$ For

\footnotetext{
${ }^{1}$ The number of pileup events would be larger than 200 if the same luminosity was achieved with $20 \mathrm{MHz}$ operation, which represents a much more demanding condition for the detectors.
} 
such scenario, CMS will eventually collect up to $3000 \mathrm{fb}^{-1}$, after several years of operation, and its tracking system will have to be improved in terms of radiation resistance, readout granularity and ability to contribute information to the Level-1 trigger. The whole tracker will have to be replaced at the beginning of the next decade: this upgrade will involve the outer strip tracker, as well as a further upgrade of the inner pixel detector.

The implementation of the trigger functionality has been studied thus far in the context of the outer tracker upgrade; this paper illustrates the options considered, discussing potential advantages and disadvantages.

\section{Requirements for the strip tracker upgrade}

The upgraded tracker will have to provide improved tracking performance in a more challenging environment, while producing at the same time fast information for the Level-1 trigger. The basic requirements can be summarized as follows:

(i) Robust tracking in operation with up to $200 \div 250$ collisions per bunch crossing in the worstcase scenario of $20 \mathrm{MHz}$ operation (to be compared to the original LHC design figure of 20 collisions per crossing); this can be achieved by maintaining the occupancy at the level of a few percent, which requires increased granularity.

(ii) Ability to provide satisfactory performance up to an integrated luminosity of about $3000 \mathrm{fb}^{-1}$, to be compared with the original figure of $500 \mathrm{fb}^{-1}$; this requires the selection of more radiation hard silicon sensor material, especially for the innermost regions, as well as more stringent criteria in the qualification of electronics and mechanical assemblies.

(iii) Reduced material in the tracking volume; the material is the most severe limitation on the performance of the present tracker [2], and it is dominated by electronics and services (notably in the region between barrel and end-cap).

(iv) Ability to contribute information to the Level-1 trigger decision, in order to hold the overall rate below $100 \mathrm{kHz}$, without compromising the physics performance of CMS. The trigger requirement is discussed in more detail below.

\subsection{Tracking information at Level-1}

The event filtering at Level-1 becomes substantially more challenging at high luminosity, not only because the rate of events passing a given selection scales with the instantaneous luminosity, but also because the performance of selection algorithms degrades with increasing pile-up. For example, the single muon Level-1 rate has an irreducible tail due to poorly measured tracks that are compatible with straight trajectories, and are therefore not removed even by increasing the $\mathrm{p}_{T}$ threshold: such an effect is aggravated at high luminosity by accidental coincidences. In the High-Level Trigger, where the information from the tracker is also added, the reconstruction is substantially improved and the rate of muon candidates follows closely the generator rate. Some improvements are expected with the "Phase-1" trigger upgrade (e.g. including the $4^{\text {th }}$ RPC and CSC stations, now under construction, will allow to request 3 out of 4 points for a muon candidate), which will yield an acceptable rate for luminosities up to about $2 \times 10^{34} \mathrm{~cm}^{-2} \mathrm{~s}^{-1}$, but the rate 

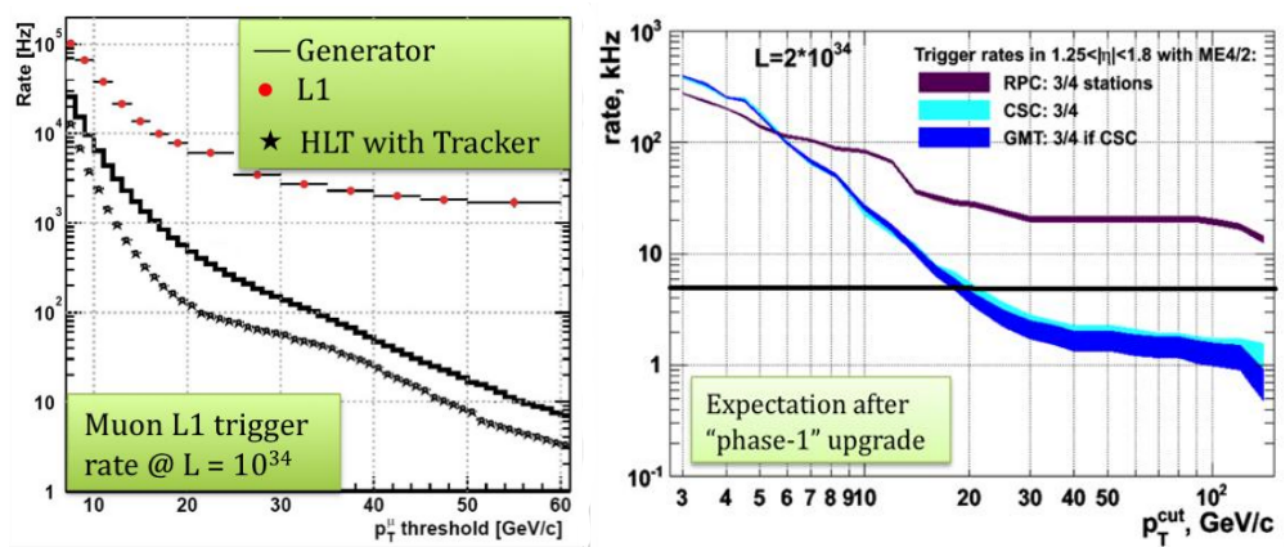

Figure 1. Expected Level-1 single muon rate (left) as a function of threshold for a luminosity of $10^{34} \mathrm{~cm}^{-2} \mathrm{~s}^{-1}$, in the present system; the rate becomes almost flat for high $\mathrm{p}_{T}$ cuts, due to poorly reconstructed tracks. The High-Level Trigger rate, instead, follows closely the generator rate, thanks to the use of tracking information. With the planned "Phase-1" upgrade of the trigger system (right), the performance of the Global Muon Trigger will be improved, achieving a rate of $5 \mathrm{KHz}$ for a target threshold of $20 \mathrm{GeV} / \mathrm{c}$ at $2 \times 10^{34} \mathrm{~cm}^{-2} \mathrm{~s}^{-1}$. At higher luminosities further improvements are needed.

will saturate again the available quota for higher luminosities (see also figure 1). Hence, a possible option to investigate is to anticipate the use of tracking information in the Level-1 selection, along the lines of what is done today in the HLT.

A similar problem is present in the single electron trigger, where high pile-up reduces the rejection power of isolation cuts on the calorimeter clusters.

\section{Implementation of the tracking trigger}

Two main architectures can be considered for the implementation of tracking trigger.

In the "push path", the tracker provides Level-1 information that is combined with calorimeter and muon trigger data (with finer granularity than presently employed) to form "physics objects" that are transmitted to the Global Trigger. In the "pull path", the present Level-1 calorimeter and muon triggers are used to produce a "Level-0" (within the current Level-1 latency of about $3 \mu \mathrm{s}$ ), that is used to request tracking information from specific "regions of interest", at an expected rate of about $1 \mathrm{MHz}$; the tracking information would then be used to form a new combined Level-1 trigger, within the remaining latency of $3 \mu \mathrm{s}$.

Current developments are focussed on the push path, which maintains the present CMS trigger architecture. This option requires that rejection of low- $\mathrm{p}_{T}$ tracks be performed locally in the module front-end, to limit the bandwidth needed by data links. Possible implementations of local data reduction exploit the strong CMS magnetic field, as discussed below.

\subsection{Modules with $\mathbf{p}_{T}$ discrimination}

The requirement for the module design is to reject locally signals from low- $\mathrm{p}_{T}$ particles, which are not interesting for the Level-1 reconstruction: rejecting particles with $\mathrm{p}_{T}$ below $1-2 \mathrm{GeV}$ reduces the bandwidth requirements by one order of magnitude or more. 

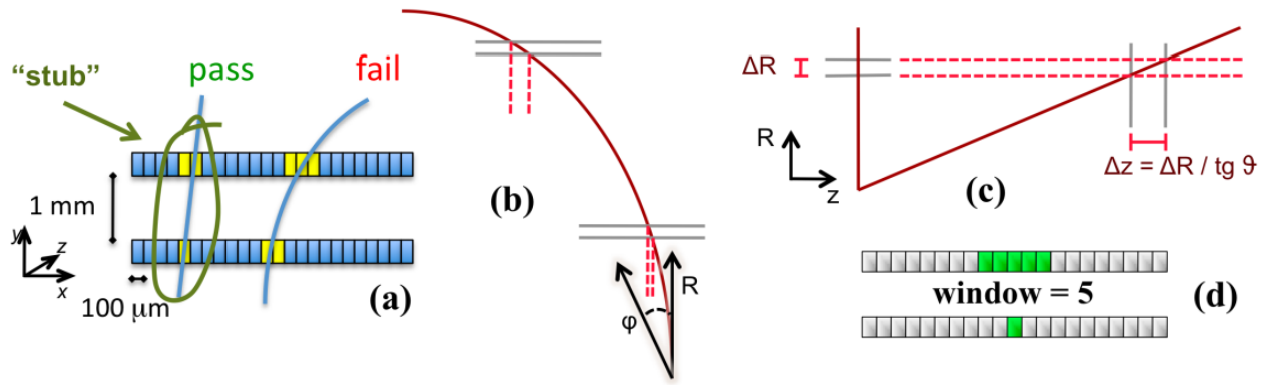

(c) $\Delta z=$

window $=5$

(d)

Figure 2. Correlation of signals in closely-spaced sensors allows to reject low- $\mathrm{p}_{T}$ particles (a). The same transverse momentum corresponds to larger distance between the two signals at large radii, for same sensor spacing (b). In end-cap configuration, a larger spacing between the sensors is needed to achieve the same discriminating power as in the barrel at the same radius (c). The acceptance window (d) can therefore be tuned at the same time as the sensors spacing to achieve the desired $\mathrm{p}_{T}$ filtering in the different regions of the detector.

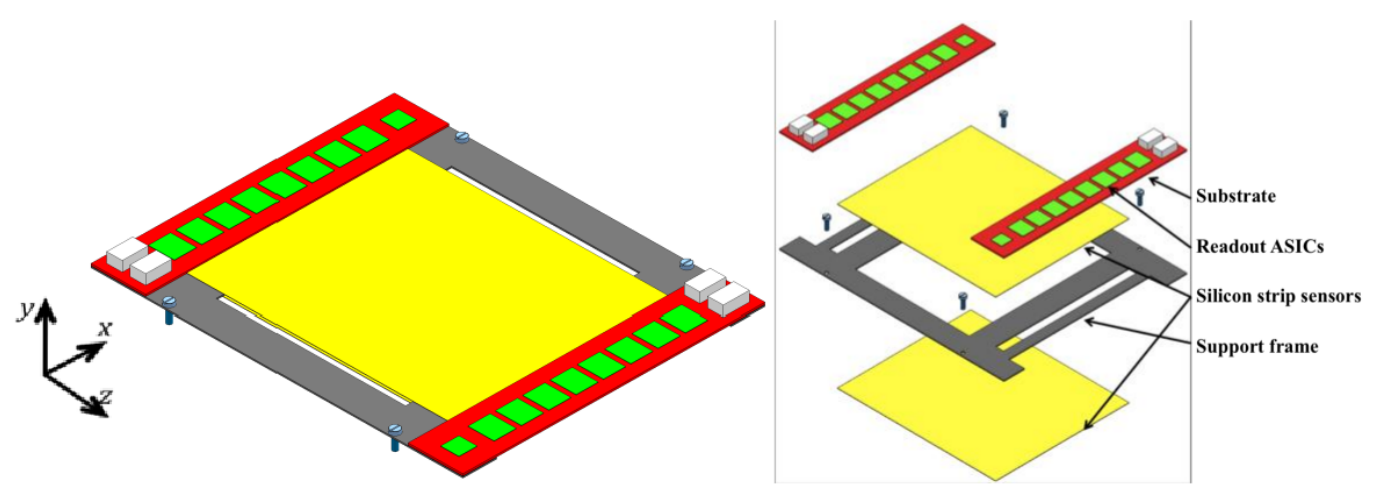

Figure 3. Model of a " $2 S$ module", made of 2 Strip sensors read out at the edges by a common set of ASICs. The connection between the sensors and the substrate carrying the ASICs is implemented through wirebonds. A lightweight frame provides support and cooling to sensors and electronics, featuring a "window" to allow wirebonding on both sides of the substrate.

The basic concept consists of correlating signals in two closely-spaced sensors: the distance between the hits in the $\mathrm{x}-\mathrm{y}$ plane is correlated with the particle $\mathrm{p}_{T}$, allowing the $\mathrm{p}_{T}$ discrimination to be made. A pair of hits that fulfils the selection cut is called a "stub", and its coordinates are sent out for the Level-1 processing (see sketches of figure 2). For a given $\mathrm{p}_{T}$, the distance between the hits forming the stub is larger at larger radii; moreover, if the module is placed in end-cap configuration, the same discriminating power is obtained with a larger spacing between the two sensors, compared to a barrel module placed at the same radius. The effective $\mathrm{p}_{T}$ cut provided by the modules in the different locations can be optimized by tuning both sensor spacing and acceptance window. The options under study to realize modules with $\mathrm{p}_{T}$ discrimination capability (" $\mathrm{p}_{T}$ modules") are discussed below. 

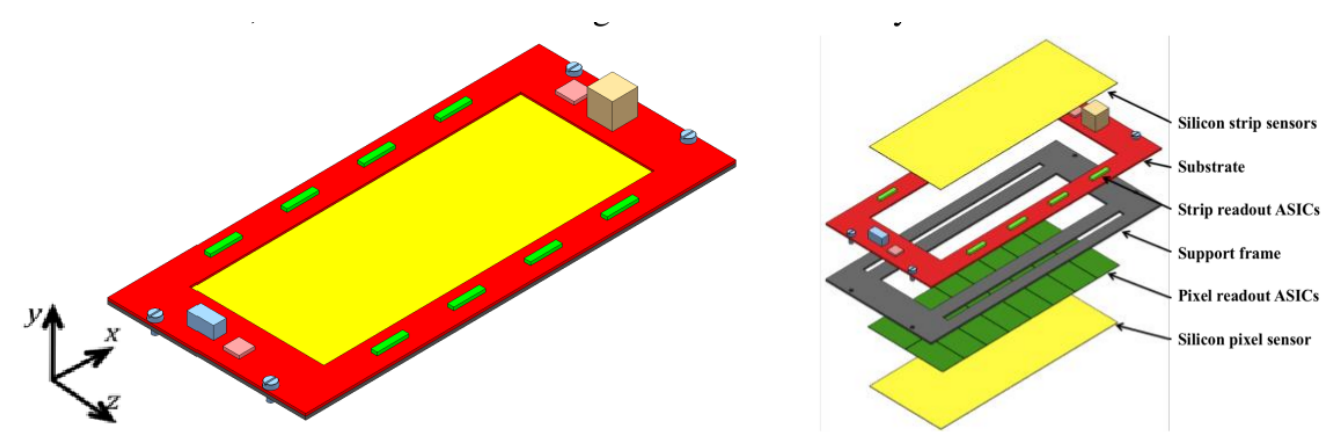

Figure 4. Model of a "PS module". Assembly and connectivity follow the same logic as for the 2S module. In this sketch a single substrate (in red) serves the whole module, carrying the front-end ASICs for the strip sensor, and all the auxiliary electronics for powering and data transmission.

\subsection{The "2S" module}

The simplest $\mathrm{p}_{T}$ module assembly is a sandwich of two strip sensors read out at the edges by the same set of front-end ASICs that implement the correlation logic. In the model shown in figure 3, two sensors of about $10 \times 10 \mathrm{~cm}^{2}$ are mounted on a mechanical structure that provides support and cooling, and connected at the edges on the two sides of a high-density substrate carrying the ASICs. Sensors and substrate are connected with wirebonds, while the ASICs can be bump-bonded onto the substrate. The strip length has to be half of the module size, hence about $5 \mathrm{~cm}$, and a suitable pitch would be $90 \mu \mathrm{m}$, corresponding to $2 \times 1024$ channels per sensor.

This concept results in a lightweight assembly that can be realized with commercial interconnection technologies. The estimated power consumption for the readout electronics, including the correlation logic, is below $2 \mathrm{~W}$, comparable to the lowest values in the present tracker. The main limitation of this type of module is the lack of segmentation in the $\mathrm{z}$ direction: in order to implement effective isolation cuts on calorimeter clusters, the Level-1 tracks must also have a reasonable precision in the $\mathrm{z}$ coordinate. In addition, the relatively long strips limit the use of this module in the radial region above $50 \mathrm{~cm}$, because of occupancy.

\subsubsection{The "PS" module}

To overcome the limitations of the $2 \mathrm{~S}$ module, another $\mathrm{p}_{T}$ module concept is under study, based on the assembly of one strip and one pixel sensor. The module has an area of approximately $5 \times 10$ $\mathrm{cm}^{2}$. The strip sensor is segmented into $2 \times 1024$ strips, making them approximately $2.5 \mathrm{~cm}$ in length. The individual pixels on the pixel sensor are approximately $1.5 \times 0.1 \mathrm{~mm}^{2}$ in size (see figure 4). The shorter module dimension along the $\mathrm{z}$ coordinate is driven by the need to cover the entire length with two pixel chips, while the shorter strips make the module suitable for operating in regions with higher particle densities. As for the $2 \mathrm{~S}$ module, strips are read out at the edges, and the connectivity between the top and bottom sensors is realized through wirebonds on the two sides of the substrate. In this case, the correlation logic is implemented in the pixel ASIC. A detailed discussion of this module can be found in [3].

Compared to the $2 \mathrm{~S}$ module, this concept offers a sufficiently precise measurement of the $\mathrm{z}$ coordinate from the pixellated sensor, while the $2.5 \mathrm{~cm}$ long strips allow the module to be used 

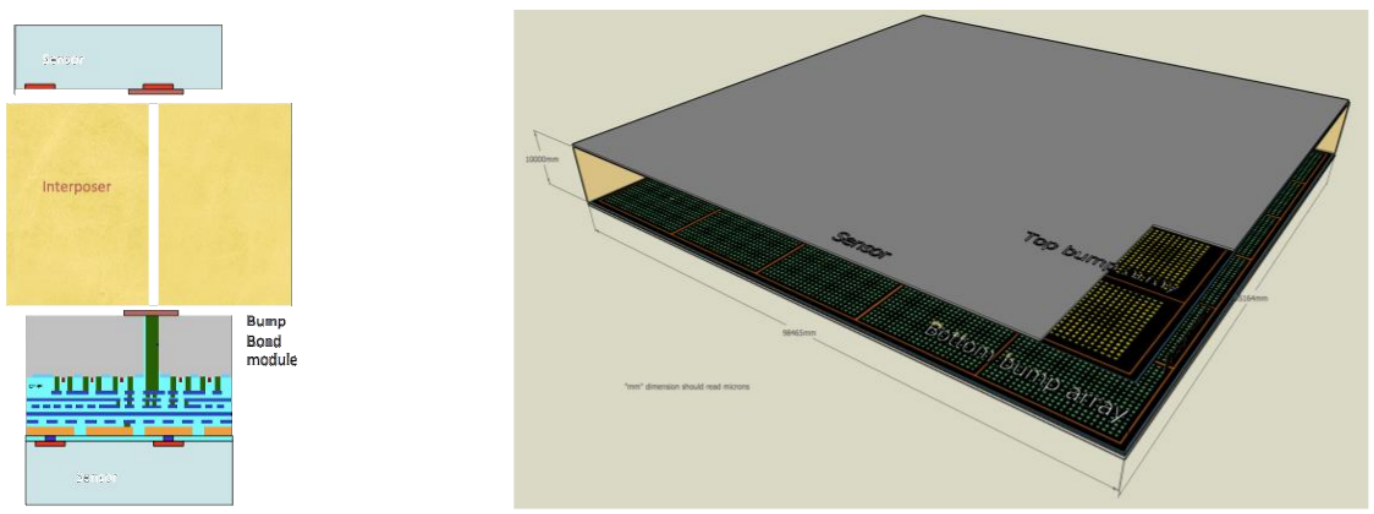

Figure 5. Sketch of the connectivity for a "VPS module" (left): a 3-d ASIC reads out at the same time the pixellated sensor and, through analogue paths in the interposer, the short-strip sensor on the other side. The interposer defines the spacing between the two sensors, carries at the same time power lines and readout signals. A $3 \mathrm{~d}$ view of a $10 \times 10 \mathrm{~cm}^{2}$ size module is shown on the right.
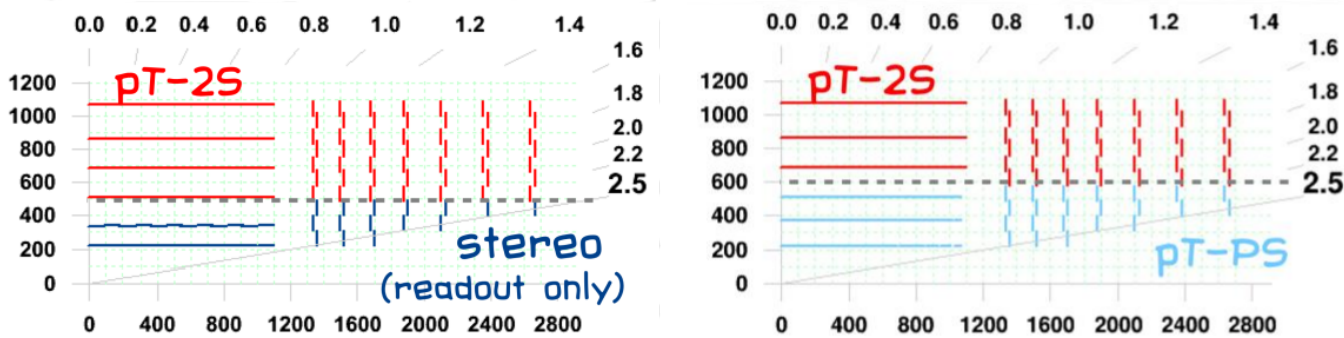

Figure 6. Left: sketch of a possible Outer Tracker layout implementing $2 \mathrm{~S}$ modules with $\mathrm{p}_{T}$ discrimination in the region $\mathrm{R}>50 \mathrm{~cm}$, complemented with stereo modules without trigger functionality in the inner part. Right: layout implementing 2S modules in the outer half of the radial range, and PS modules in the inner half.

down to $20 \div 25 \mathrm{~cm}$ radius. On the other hand the power consumption is expected to be more than $4 \mathrm{~W}$, dominated by the pixel ASICs: a four-fold increase in power density compared to the $2 \mathrm{~S}$ module, which translates to a higher estimated density of material.

\subsubsection{The "VPS" module}

A different approach to the construction of a pixel/strip $\mathrm{p}_{T}$ module is based on vertical interconnections. One 3-d chip reads out both the pixellated sensor and the short-strip sensor, connected by analogue paths through an interposer (figure 5), and implements the correlation logic. The use of vertical connectivity removes all constraints between module dimensions and sensors segmentation: such a module can in principle be made in $10 \times 10 \mathrm{~cm}^{2}$ size, and, if needed, the strip length can be reduced to further reduce the occupancy (e.g. about $1 \mathrm{~cm}$ ).

Since there is no connectivity between modules, a larger module size offers advantages for the integration of hermetic surfaces, reducing the need of overlaps. On the other hand, feasibility, reliability and yield of vertical interconnections for large-surface assemblies need to be verified, considering the demanding operating conditions inside CMS. In addition, in this concept a $1 \mathrm{~mm}$ 


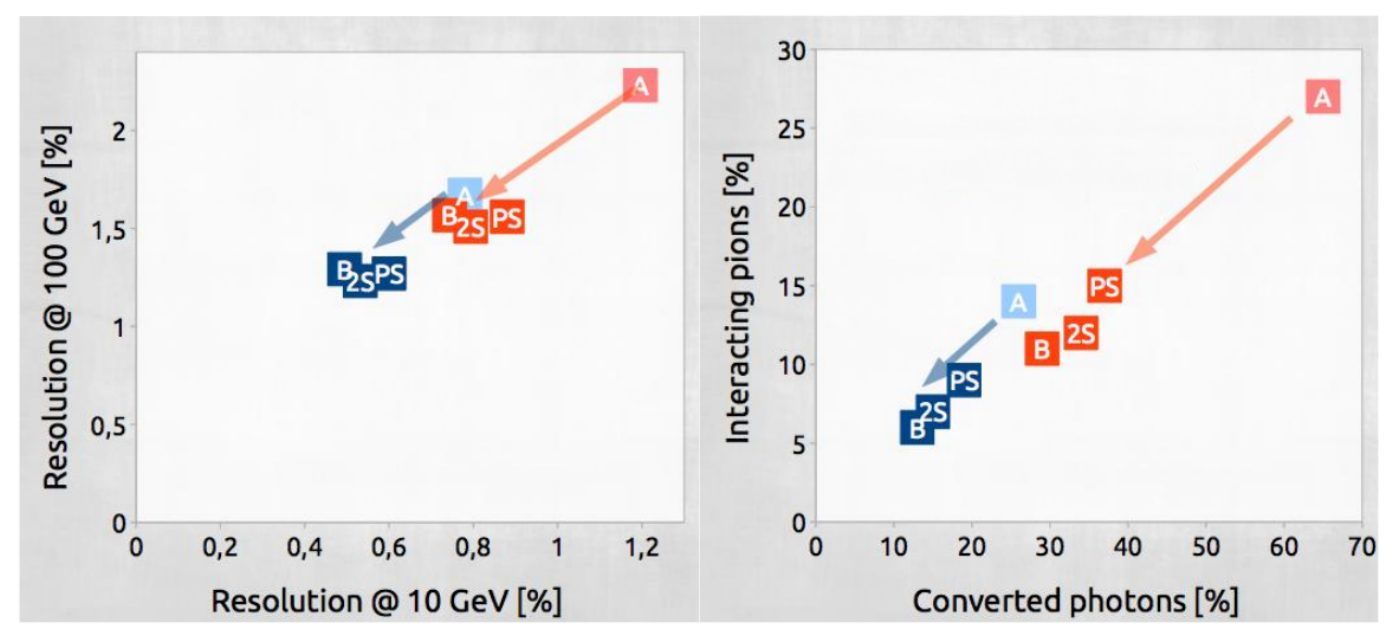

Figure 7. Comparison of momentum resolution for $10 \mathrm{GeV}$ and $100 \mathrm{GeV}$ tracks (left), and fraction of interacting pions and converted photons (right) for 4 tracker layouts, namely A: the present tracker, B: upgraded tracker without trigger functionality (not discussed in this paper), 2S: layout of figure 6 (left), PS: layout of figure 6 (right). Blue and orange markers correspond to the central and intermediate rapidity regions, respectively, as defined below in figure 8 .

thick interposer covers the entire surface of the module, defining the spacing between the two sensors, providing the top-to-bottom connectivity and carrying power lines and readout signals: the development of a lightweight interposer with the necessary electrical and mechanical properties is a key issue to realize a module with an acceptable material density.

\section{Evaluation of tracker options and geometries}

A dedicated standalone software package ("tkLayout") has been developed to quantitatively evaluate the different options and geometries for the tracker upgrade. The software generates detector layouts starting from a reasonably small set of parameters, and also provides simple and flexible implementation of material densities for active and inactive volumes, including the routing of the services. The software calculates, as a function of pseudorapidity, the expected tracking precision and the performance potential for the Level-1 track reconstruction, as well as the fraction of converted photons and interacting pions. In addition, it generates summary statistics such as number of modules, readout channels, power consumption, total weight and many others. The package has been validated by modelling the present tracker, demonstrating an excellent agreement between the predicted performance and the one measured from the real detector. Some examples of the studies that have been carried out are shown below.

Sketches of two layouts that have been modelled are shown in figure 6: the one on the left implements $2 \mathrm{~S}$ modules in the outer part, down to $\mathrm{R} \approx 50 \mathrm{~cm}$, complemented with stereo modules without trigger functionality (not discussed in this paper) in the region between 20 and $50 \mathrm{~cm}$; the layout on the right implements modules with $\mathrm{p}_{T}$ discrimination in the whole tracking volume, $2 \mathrm{~S}$ modules in the outer half of the radial range, and PS modules in the inner half. 


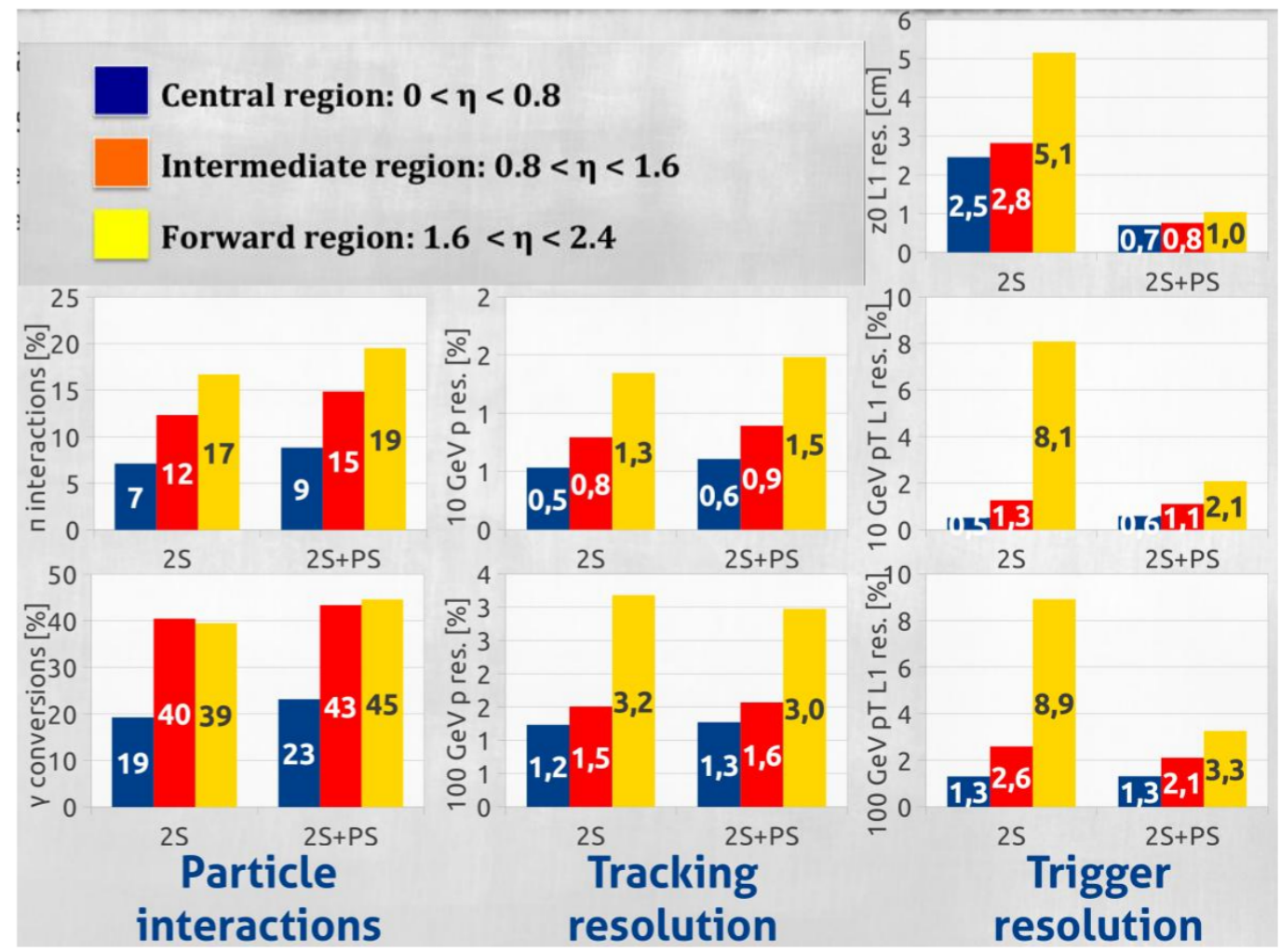

Figure 8. Comparison of the expected performance of the two layouts shown in figure 6 , in three rapidity ranges. The two layouts have very similar momentum resolution, the layout with PS modules has somewhat larger probability of particle interaction, but the performance potential for the Level-1 reconstruction is substantially improved, notably the momentum resolution in the forward region, and the z0 resolution in the whole rapidity range. The z0 resolution is expected to be relevant to be able to discriminate between the different collision points.

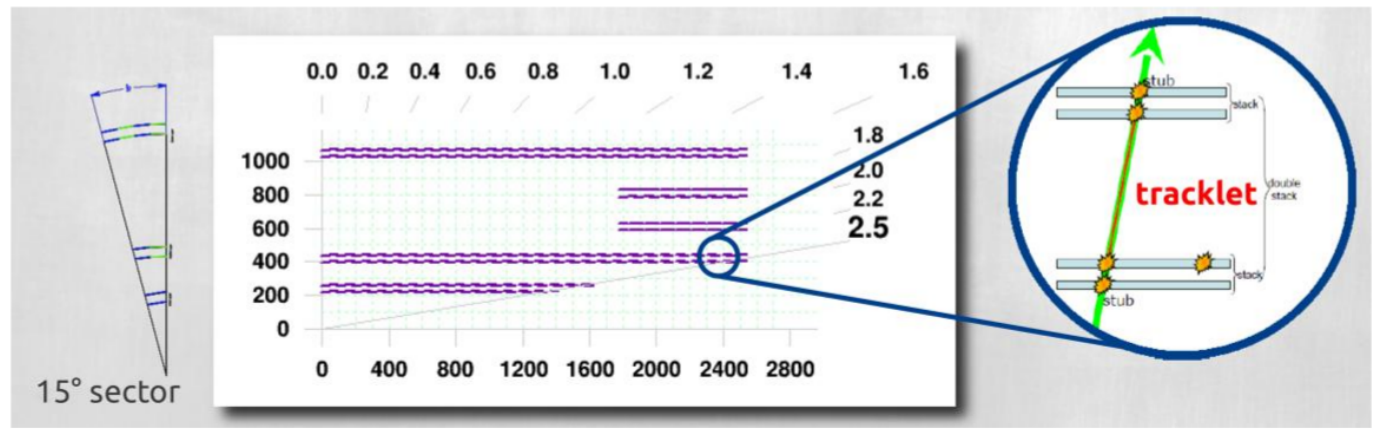

Figure 9. Hierarchical processing of stub coordinates. In a detector geometry where layers are arranged in pairs ("double-stack" geometry), pairs of stubs are first combined to form "tracklets" which are then used as seeds for the Level-1 track finding. The modularity in $\phi$ is tuned to have self-contained $15^{\circ}$ sectors, in order to limit the amount of cabling at the back-end, and mitigate the complexity of the combinatorial problem to be solved. The concept has been studied in barrel geometry. 
In figure 7 the expected tracking precision and fraction of interacting particles for each of these two layouts are compared with the present tracker and with an upgraded tracker without trigger functionality (not discussed in this paper). The new technologies that will be used offer the possibility of substantial improvements in tracking precision and reduced fraction of interacting particles (A vs B). The performance of the layout implementing $2 \mathrm{~S}$ modules is very close to "B", showing that the use of $2 \mathrm{~S}$ modules with trigger functionality has minimal impact on the tracker performance. A larger difference is observed for the layout with PS modules, because of the larger amount of material mostly due to the higher power density. A more detailed and quantitative comparison between the two layouts is given in figure 8: the use of PS modules substantially improves the performance potential for the Level-1 reconstruction.

\section{Tracking trigger: from stubs to tracks}

The coordinates of stubs selected by the $\mathrm{p}_{T}$ modules are sent to the trigger electronics at the backend, where they have to be combined to form Level-1 tracks. The target is to reconstruct with high efficiency tracks of particles with $\mathrm{p}_{T}>2 \mathrm{GeV}$, which should allow to perform isolation cuts on calorimeter clusters. The two concepts considered so far are briefly discussed below.

\subsection{Hierarchical processing in FPGA}

The possibility of processing the coordinates of the selected stubs in a dedicated set of FPGAs has been studied in some detail, using a detector geometry optimized for trigger reconstruction. Layers are arranged in closely-spaced pairs ("double-stack" geometry) in order to mitigate the combinatorial problem, and pairs of stubs are first combined to form "tracklets", that have sufficient precision to extrapolate to the next double stack (sketches in figure 9). Tracklets and remaining unassociated stubs are then combined to form Level-1 tracks. The geometry is also tuned to have well-defined sectors in the $\phi$ view. Good progress has been made in exploring this option, with barrel geometry.

\subsection{Parallel processing in Associative Memories}

Parallel processing in Associative Memories is considered as an alternative approach, which could allow stub coordinates to be processed in a generic detector geometry, by comparing the collected coordinates with pre-stored patterns that correspond to high- $\mathrm{p}_{T}$ tracks. This approach was successfully used in the CDF trigger, and is being considered for the ATLAS trigger upgrade [4]. However the application in CMS would be of unprecedented size and complexity, and feasibility studies have just started.

\section{Conclusions}

Module concepts that offer the potential for precise tracking at Level-1 in the planned upgrade of the CMS Tracker have been discussed. Modelling studies at the detector system level show that the trigger functionality can be implemented with an acceptable impact on tracking performance. Compared to the present CMS Tracker, an improvement between $30 \%$ and 50\% in momentum 
resolution seems to be achievable, while the rate of particles (hadrons or photons) interacting in the detector material can be reduced by almost a factor of two.

The study of options to process the trigger information to form Level-1 tracks has also started. The set of software tools developed to evaluate the different options in terms of the expected detector performance will be an asset to achieve an optimal tracker design.

\section{Acknowledgments}

I wish to thank the organizers for a very interesting workshop and a very pleasant stay in Vienna.

\section{References}

[1] W. Bertl, CMS pixel upgrade, PoS (VERTEX 2011) 019.

[2] G. Sguazzoni, Performance of the CMS silicon Tracker, PoS (VERTEX 2011) 013.

[3] A. Marchioro, A hybrid module architecture for a prompt momentum discriminating tracker at SLHC, POS (VERTEX 2011) 037.

[4] J.M. Tuggle, ATLAS FTK: fast track trigger, PoS (VERTEX 2011) 040. 